\title{
Enhanced Recovery After Surgery Multi-Modality Pain Regimen Performs Similar to PRN Narcotics on Outcomes and Pain Control After Cardiac Surgery: A Quality Improvement Project
}

\author{
David Blitzer ${ }^{1}$, Chad T. Blackshear ${ }^{2}$, Jameika Stuckey ${ }^{3}$, Leslie Kruse ${ }^{3}$, Lawrence L. \\ Creswell $^{4}$, Seth Lirette ${ }^{2}$, and Hannah Copeland ${ }^{5}$ \\ ${ }^{1}$ Columbia University Department of Surgery \\ ${ }^{2}$ Fulcrum - Jackson MS \\ ${ }^{3}$ University of Mississippi Department of Pharmacy Practice \\ ${ }^{4}$ University of Mississippi Medical Center Department of Surgery Division of Cardiac \\ Surgery - Jackson Mississippi \\ ${ }^{5}$ Lutheran Hospital
}

December 25, 2021

\begin{abstract}
Background: While enhanced recovery after surgery (ERAS) pathways have been successfully applied for cardiac surgery, there has been limited research directly comparing ERAS protocols to ad hoc narcotic use after surgery. We hypothesized that a standardized ERAS protocol would provide similar pain management and psycho-emotional outcomes while decreasing the use of opioids in the hospital and after discharge. Methods: As part of a 7-month quality improvement project, cardiac surgery patients on a fast tracked to extubate pathway were assigned PRN narcotic pain management for 3 months ( $\mathrm{n}=49$ ). After a 1-month ERAS protocol optimization period, a separate group of patients were given the ERAS protocol ( $\mathrm{n}=34$ ). Clinical outcomes were gathered, and participants completed a quality of recovery survey that allowed for the assessment of pain and symptom control at 4 time-points post-surgery. Results: Among 83 participants, $66 \%$ were male and the mean age was 53 years. There were no differences in patient characteristics between PRN and ERAS groups (all p >0.244). There were no differences between ERAS and PRN groups for surgery characteristics (all p >0.060), inpatient outcomes (all p $>0.658$ ), or after-discharge outcomes (all p>0.397). Furthermore, across all time-point comparisons, there were no supported differences in patient-reported outcome and pain control between the ERAS and PRN narcotic groups (all p>0.075). Conclusions: An ERAS protocol demonstrated similar patient outcomes and pain control to traditional opioid use for postoperative cardiac surgery patients. Further research is recommended to further confirm the results of this study.
\end{abstract}

\section{Introduction}

Enhanced recovery after surgery (ERAS) protocols are being used in various surgical areas in an effort to reduce the use of opioids while responding to the trauma of surgery and postoperative pain. This has become increasingly important in the wake of the opioid crisis and the confirmed role that surgeons can play . [1-3] Cardiac surgeons are not without fault in this crisis, with some studies demonstrating that nearly $10 \%$ of cardiac surgery patients go on to develop persistent opioid requirements after cardiac surgery [4] Indeed, ERAS protocols are highlighted for their pain managements strategies which the do not rely on opioids, but the benefits extend beyond pain management. These protocols have demonstrated improvement in length of stay and overall postoperative complications in multiple surgical specialties.[5,6] Hirji et al developed a list of objective data elements which could be collected to demonstrate the benefits of ERAS 
protocols for cardiac surgery and standardize benchmarks across hospitals.[7] A brief commentary noted the benefits of ERAS protocols for cardiac surgery with early extubation, potentially even in in the operating room immediately after surgery.[8] Williams et al noted in a 1-year study that ERAS protocols for cardiac surgery programs had significantly improved perioperative outcomes including decreased intensive care unit (ICU) stay and hospital length of stay (LOS), decreased incidence of gastrointestinal (GI) complications, and decreased utilization of narcotic pain regimens. [9] Pain management and opioid sparing strategies are only a portion of a comprehensive ERAS protocol. The benefits of these protocols go beyond pain control and are multi-dimensional in their aim to improve the patients' surgical experience. Adequate pain control may lead to patient satisfaction, overall improved attitude towards the postoperative experience, and increased willingness to participate in postoperative care (eg, physical therapy etc). Currently, the ERAS studies in cardiac surgery have focused on many aspects of improving the postoperative course of patients, including faster time to extubation, decreased length of stay, and others. We developed an ERAS protocol as a quality improvement initiative and sought to evaluate its effect regarding pain control. We hypothesized that with a multi-modality pain regimen and limited narcotics administration, patients' pain would be well controlled after cardiac surgery, resulting in improved patient satisfaction.

\section{Methods}

\section{Patient Population}

The current study was a single-center, IRB approved, prospective, sequentially allocated, non-randomized quality improvement trial. Any patient undergoing either elective or urgent cardiac surgery deemed appropriate for fast track to extubate was eligible for inclusion in the study, including both PRN and ERAS arms. Any patient undergoing non-emergent surgery was eligible for the fast track to extubate pathway.

Data were collected over a 7 -month period in 2019. For the first 3 months of the study, any eligible participant received the traditional PRN narcotic regimen which included medications such as hydrocodone/acetaminophen and oxycodone/acetaminophen PRN. These patients are denoted as the "PRN" group. The ERAS protocol was then introduced for all eligible patients at a single medical center. Following a 1-month protocol optimization period, eligible patients were treated using the ERAS pain management protocol over the subsequent 3 months. These participants are denoted as the "ERAS" group. The full preand post-operative ERAS protocols are shown in Figures $\mathbf{1}$ and $\mathbf{2}$.

\section{Clinical Characteristics}

Clinical metrics assessed included: aortic cross-clamp time and cardiopulmonary bypass time during the operation, ICU and in-hospital LOS, rates of postoperative atrial fibrillation, wound infection, stroke, prolonged ventilation, and 30-day mortality and readmission rate.

\section{Quality of Recovery}

Participants were given a 40-item quality of recovery survey (QoR-40) at four time points during the study: (i) at 48 hours post-extubation, (ii) at transfer from ICU to non-ICU, (iii) at hospital discharge, and (iv) at a 2-week in-person clinic follow-up visit. We assessed various dimensions of recovery via item-level symptom reports of pain ( 7 questions), physical comfort (8 questions), emotional state (6 questions), and psychological support (1 question).[10] Each participant was asked "Have you had any of the following in the last 24 hours?" They were then asked to grade these symptoms on a 5-point Likert scale: "None of the time", "Some of the time", "Usually", "Most of the time", and "All of the time". (Instrument included in Supplementary Materials).

\section{Statistical Analysis}

Summary statistics were calculated using standard methods. Multivariable mixed effects ordered logistic models were employed using self-reported frequencies across symptom categories. The primary modeling framework included an interaction of peri-surgical time point and pain management protocol, adjusted for age, race, gender, body-mass index (BMI), history of intravenous (IV) drug use, and use of pain medications 
at home around the surgical window. Graphs were drawn using marginal estimates from fully adjusted models. Due to limited data in some response categories, results were presented for participants reporting "none" or "some" symptom occurrences at each time point. Furthermore, for some outcomes, absence of variation in the data would not allow for model convergence, so these outcomes are omitted from reported results. There was no reason to assume missing data were not missing at random (NMAR). The missing at random (MAR) assumptions of the mixed models utilized in analyses were deemed valid. All statistical analyses were completed using Stata v16.1.

\section{Results}

There were 83 participants, 49 in the PRN narcotic group and 34 assigned to ERAS. Sixty-six percent were male and the mean age was 53 years (Table 1 ). There were no differences in patient characteristics between PRN and ERAS groups; this includes no significant different in age $(54.37 \pm 13.56$ PRN vs $51.76 \pm 14.83$; $\mathrm{p}=0.41)$, history of IV drug use $(\mathrm{n}=4 \mathrm{PRN}$ vs. $\mathrm{n}=2 \mathrm{ERA} ; \mathrm{p}=0.69)$ or prevalence of prior pain medication prescriptions ( $\mathrm{n}=11$ PRN vs $\mathrm{n}=10 \mathrm{ERA} ; \mathrm{p}=0.47)$ The type of operations included was heterogeneous and included coronary artery bypass grafting $(\mathrm{CABG})$ alone $(\mathrm{n}=27 ; 32 \%)$, valve surgery alone $(\mathrm{n}=15 ; 18 \%)$, CABG + valve $(n=3 ; 4 \%)$, CABG + other $(n=2 ; 2 \%)$, Valve + other $(n=13 ; 16 \%)$, and other procedures $(\mathrm{n}=23 ; 28 \%)$, with the vast majority of these procedures consisting of thoracic aortic repair. All procedures were performed via median sternotomy. Surgical characteristics are described in Table $\mathbf{2}$. There was no difference in hospital mortality ( $\mathrm{n}=1$ PRN vs $\mathrm{n}=1 \mathrm{ERA} ; \mathrm{p}=0.79$ ), hospital length of stay (16.8 days PRN vs. 15.35 days ERAS; $\mathrm{p}=0.66$ ), or ICU length of stay (6.88 days PRN vs 6.88 days ERAS; $\mathrm{p}=0.99$ ). There was a trend toward significant for operative cardiopulmonary bypass times (145.41 minutes PRN vs. 114.85 minutes ERAS; $\mathrm{p}=0.06$ ) but no difference in aortic cross clamp times (97 minutes PRN vs 75.82 minutes ERAS; $\mathrm{p}=0.11$ ).

There were no supported differences in patient-reported outcome and pain control between the ERAS protocol and traditional narcotic PRN-only pain medications (Table 3 ). As an illustrative example of patientreported trajectories at the 4 time points, Figure 3shows percentage of patients reporting headache either none of the time (green) or some of the time (orange) for both ERAS (solid lines) and PRN (dashed lines). For this particular outcome, headache remained stable across time, with those on the ERAS protocol displaying a higher proportion of having no headache. Trajectory relationships for other outcomes are shown in Figures 4 and $\mathbf{5}$. Low-level presence of almost all patient reported adverse outcomes and pain was observed across the full battery of symptoms reported (Figures 3-5 ), with the exception of moderate pain being reported in equal numbers across all time points.

\section{Conclusions}

Despite lacking statistical significance, our data suggest an overall reduction in reported symptoms associated with the ERAS protocol at hospital discharge, compared to ICU transfer (Table $3 ; 10 / 16$ ORs $<1$ ). Conversely, the data also suggest an overall increase in symptom reporting associated with the ERAS protocol at the 2 -week outpatient visit, compared to hospital discharge (Table $3 ; 14 / 16$ ORs $>1$ ). The notable exception to this was the odds of reporting severe pain at the 2 weeks follow up vs. hospital discharge $(\mathrm{p}=0.075)$. However, this relationship can be partially explained by the very low reporting of severe pain at hospital discharge for the ERAS group. In our sample, patients rarely reported any outcome with frequency beyond "some of the time".

Surgeons have proven to be high opioid prescribers, and previously there was limited evidence to support discrete recommendations [11-13]. In general however, surgeons have been found to be over-prescribers of opioid pain medications [11]. This has prompted the proliferation and study of protocols which minimize narcotic pain medications. Few studies in the cardiothoracic literature have specifically addressed the influence of pain medication regimens, and adequacy of pain control on patient satisfaction with an ERAS protocol. Wagner et al performed a multicenter retrospective analysis on patient undergoing CABG and identified that in their study $28 \%$ of opioid naïve patients could be discharged without an opioid prescription and the vast majority of these never required an opioid prescription at follow-up [14]. Pan et al evaluated 
patients' satisfaction after elective cesarean section.[15] The study noted that patients who were treated with an ERAS protocol reported a decreased incidence of intraoperative nausea, decreased pain scores during the first 24 hours at rest, and during motion in the first 24 hours and 48 hours after surgery. They also reported that patient satisfaction was higher in the ERAS group. Li et al published a review article on the use of ERAS protocols in colorectal surgery.[16] Four of the 15 publications discussed patient satisfaction with the use of an ERAS protocol. There was no difference in patient satisfaction in 2 of these studies. Just one study noted an improvement in overall patient satisfaction scores. Li et al concluded that patient satisfaction was not worse with an ERAS protocol, which is similar to the results of our study. Debono et al noted the use of an ERAS protocol in spine surgery, specifically spine fusion surgery.[17] The study noted, based on a 5-point Likert scale, that $86.5 \%$ of patients were satisfied or very satisfied with overall care. Rege et al also noted improved pain scores with an ERAS protocol in laparoscopic donor nephrectomy.[18] Echeverria-Villabolos et al conducted a study to evaluate ERAS protocol to reduce postoperative opioid use.[19] The study noted that an effective multi-modality pain regimen that was narcotic-free, including medications such as nonsteroidal anti-inflammatory drugs (NSAIDs), acetaminophen, gabapentin, regional anesthetic medications, etc, was associated with decreased narcotic use in the postoperative setting.

Limited studies exist that evaluate patient satisfaction in the postoperative environment with regard to pain control in all of the surgical literature. This study evaluates the use of a multi-modality minimal narcotic pain regimen for cardiac surgery patients. When compared to a more traditional narcotic based pain regimen, patients' satisfaction demonstrated no difference. This study emphasizes in addition to other ERAS studies in cardiac surgery, that patients' recovery quality can be maintained and potentially benefit from a minimal narcotic regimen.

There are some limitations in our study to consider. The analysis sample was recruited via mutually exclusive pre-defined recruitment windows for each treatment group, potentially contributing to some degree of bias among the study characteristics between the treatment groups. The limited time window for data collection paired with the patient throughput restricted the sample further than desired leaving some potentially clinically meaningfully differences undetected.

Future studies would benefit from additional assessment for pain intensity, such as the visual analogue scale (VAS) or the numeric rating scale (NRS). Subgroup analyses for surgical approach would uncover potential confounders to the performative evaluation of the ERAS protocol.

In conclusion, we have demonstrated the potential for the ERAS protocol to provide equivalent pain and psychological outcomes compared to PRN opioids for cardiac surgery patients. These results need to be confirmed with rigorously designed randomized clinical trials, including multiple sites and increased patient recruitment. With the potential to drastically reduce the amount of opioids in the community, this research should be a high priority.

\section{Tables}

Table 1. Patient Characteristics at Enrollment Table 1. Patient Characteristics at Enrollment

\section{Characteristics}

$(\mathrm{n}=83)$

$(\mathrm{n}=49)$

$(\mathrm{n}=34)$

Age (years)

Ethnicity

Race

\section{PRN \\ ERAS \\ p-value}

Hispanic or Latino

Not Hispanic or Latino

Unknown / Not Reported

American Indian/Alaska Native

Asian

Native Hawaiian or Pacific Islander

Black or African American
Overall

$53.30(14.0$

$3(4 \%)$

$77(93 \%)$

$3(4 \%)$

$2(2 \%)$

$0(0 \%)$

$0(0 \%)$

$40(48 \%)$ 
Table 1. Patient Characteristics at Enrollment Table 1. Patient Characteristics at Enrollment

White

Male $(\mathrm{Y} / \mathrm{N})$

Height $(\mathrm{cm})$

Weight $(\mathrm{kg})$

BMI $\left(\mathrm{kg} / \mathrm{m}^{2}\right)$

History of IV Drug Use (Y/N)

Prescription Pain Medication at Home (Y/N)

Cells represent: mean (SD) or n (\%)
More Than One Race

Unknown / Not Reported

$38(46 \%)$

$1(1 \%)$

$2(2 \%)$

$55(66 \%)$

$173.88(9.3$

$89.22(20.8$

$29.45(6.33$

History of IV Drug Use (Y/N)

$6(7 \%)$

Prescription Pain Medication at Home (Y/N)

$21(25 \%)$

BMI, body-mass index; IV, intravenous

Table 2. Surgical characteristics

\section{Characteristics}

$(\mathrm{n}=83)$

$(\mathrm{n}=83)$

$(\mathrm{n}=49)$

$(\mathrm{n}=34)$

$(\mathrm{n}=34)$

In-hospital mortality $(\mathrm{Y} / \mathrm{N})$

In-hospital stroke $(\mathrm{Y} / \mathrm{N})$

Postoperative atrial fibrillation $(\mathrm{Y} / \mathrm{N})$

Hospital length of stay (days)

ICU length of stay (days)

Ventilator time (days)

30-day mortality $(\mathrm{Y} / \mathrm{N})$

30-day wound infection $(\mathrm{Y} / \mathrm{N})$

30-day readmission $(\mathrm{Y} / \mathrm{N})$

Operative cardiopulmonary bypass time (minutes)

Operative aortic cross-clamp time (minutes)

Follow-up reoperation $(\mathrm{Y} / \mathrm{N})$

Cells represent: mean (SD) or n (\%) ICU, intensive care unit.

Table 3. Effect modification of ERAS protocol for various symptoms across study window Have you had any of the following in the last 24 hours?

vs 48-hrs Post-Extubation

vs 48-hrs Post-Extubation

vs ICU Transfer

vs ICU Transfer

vs ICU Transfer

vs Hospital Discharge

vs Hospital Discharge

Physical comfort

Nausea

Feeling restless

Shaking or twitching

Shivering

Feeling too cold

Feeling dizzy 
Table 2. Surgical characteristics

\section{Emotion State}

Feeling anxious

Feeling angry

Feeling depressed

Feeling alone

Had difficulty falling asleep

Pain

Moderate pain

Severe pain

Headache

Muscle pains

Backache

Cells represent: adjusted OR (95\% CI OR) p-value

Example interpretation: When returning for a 2 -week outpatient surgical follow-up visit, there was an associated $12 \%$ incre

ICU, intensive care unit.

\section{Figure Legends}

Figure 1. Details of the ERAS protocol, Pre-Op

Figure 2. Details of the ERAS protocol, Post-Op

Figure 3. Adjusted symptom probabilities (with 95\% CIs) of participants reporting none or some headaches under each protocol across peri-surgical period

Figure 4. Adjusted symptom probabilities (with 95\% CIs) of participants reporting none or some pain-related symptoms under each protocol across peri-surgical period.

Figure 5. Adjusted symptom probabilities (with $95 \%$ Cis ) of participants reporting none or some quality of life-related symptoms under each protocol across peri-surgical period

\section{References}

1. Chen LH, Hedegaard H, Warner M. Drug-poisoning deaths involving opioid analgesics: United States, 1999-2011. NCHS Data Brief, 166 (2014), pp. 1-8

2. McCarthy M. Opioid prescribing rates in US vary widely between states, CDC reports. BMJ, 349 (2014), p. 4424, 10.1136/bmj.g4424

3. Levy B, Paulozzi L, Mack KA, Jones CM. Trends in opioid analgesic-prescribing rates by specialty, U.S., 2007-2012 .Am J Prev Med, 49 (3) (2015), pp. 409-413, 10.1016/j.amepre.2015.02.020

4. Brown CR, Chen Z, Khurshan F, Groeneveld PW, Desai ND. Development of Persistent Opioid Use After Cardiac Surgery. JAMA Cardiol. 2020;5(8):889-896. doi:10.1001/jamacardio.2020.1445

5. Engelman DT, Ben Ali W, Williams JB, et al. Guidelines for Perioperative Care in Cardiac Surgery: Enhanced Recovery After Surgery Society Recommendations. JAMA Surg. 2019 Aug 1;154(8):755-766. doi: 10.1001/jamasurg.2019.1153. PMID: 31054241.

6. Thiele RH, Rea KM, Turrentine FE, et al. Standardization of care: impact of an enhanced recovery protocol on length of stay, complications, and direct costs after colorectal surgery. J Am Coll Surg. $2015 ; 220(4): 430-443$.

7. Hirji SA, Salenger R, Boyle EM, et al. Expert Consensus of Data Elements for Collection for Enhanced Recovery After Cardiac Surgery. World J Surg. 2021; 45: 917 - 925.

8. Chatterje S. Commentary: The enhanced recovery train is leaving the station - Compliance with phase of care guidelines is associated with earlier extubation and shorter length of stay as part of an enhanced recovery after cardiac surgery program. J Thorac Cardiovasc Surg. 2020 Apr;159(4):1405-1406. 
9. Williams JB, McConnell G, Allender JE, et al. One-year results from the first US-based enhanced recovery after cardiac surgery (ERAS Cardiac) program. J Thorac Cardiovasc Surg. 2019 May;157(5):18811888.

10. Myles P, Weitkamp B, Jones K, Melick J, Hensen S. Validity and reliability of a postoperative quality of recovery score: the QoR-40. Br J Anaesth 2000; 84: 11-15.

11. Thiels CA, Anderson SS, Ubl DS, et al. Wide variation and overprescription of opioids after elective surgery. Ann Surg, 266 (4) (2017), pp. 564-573, 10.1097/SLA.0000000000002365

12. Bicket MC, Long JJ, Pronovost PJ, Alexander GC, Wu CL. Prescription opioid analgesics commonly unused after surgery: a systematic review. JAMA Surg, 152 (11) (2017), pp. 1066-1071, 10.1001/jamasurg.2017.0831

13. Scully RE, Schoenfeld AJ, Jiang W, et al. Defining optimal length of opioid pain medication prescription after common surgical procedures. JAMA Surg, 2115 (2017), pp. 1-7, 10.1001/jamasurg.2017.3132

14. Wagner CM, Clark MJ, Theurer PF, Lall SC, Nemeh HW, Downey RS, Martin DE, Dabir RR, Asfaw ZE, Robinson PL, Harrington SD, Gandhi DB, Waljee JF, Englesbe MJ, Brummett CM, Prager RL, Likosky DS, Kim KM, Lagisetty KH, Brescia AA; Michigan Society of Thoracic and Cardiovascular Surgeons Quality Collaborative (MSTCVS-QC). Predictors of Discharge Home Without Opioids After Cardiac Surgery: A Multicenter Analysis. Ann Thorac Surg. 2021 Dec 9:S0003-4975(21)01842-7. doi: 10.1016/j.athoracsur.2021.10.005. Epub ahead of print. PMID: 34924190.

15. Pan J, Hei Z, Li L, et al. The Advantage of Implementation of Enhanced Recovery After Surgery (ERAS) in Acute Pain Management During Elective Cesarean Delivery: A Prospective Randomized Controlled Trial. Ther Clin Risk Manag. 2020;16:369-378.

16. Li D, Jensen CC. Patient Satisfaction and Quality of Life with Enhanced Recovery Protocols. Clin Colon Rectal Surg. 2019 Mar;32(2):138-144.

17. Debono B, Corniola MV, Pietton R, et al. Benefits of Enhanced Recovery After Surgery for fusion in degenerative spine surgery: impact on outcome, length of stay, and patient satisfaction. Neurosurg Focus. 2019 Apr 1;46(4):E6.

18. Rege A, Leraas H, Vikraman D, et al. Could the Use of an Enhanced Recovery Protocol in Laparoscopic Donor Nephrectomy be an Incentive for Live Kidney Donation? Cureus. 2016 Nov 22;8(11):e889.

19. Echeverria-Villalobos, Marco; Stoicea, Nicoleta; Todeschini, Alexandre B, et al. Enhanced Recovery After Surgery (ERAS). The Clinical Journal of Pain: March 2020; 36(3): 219-226.

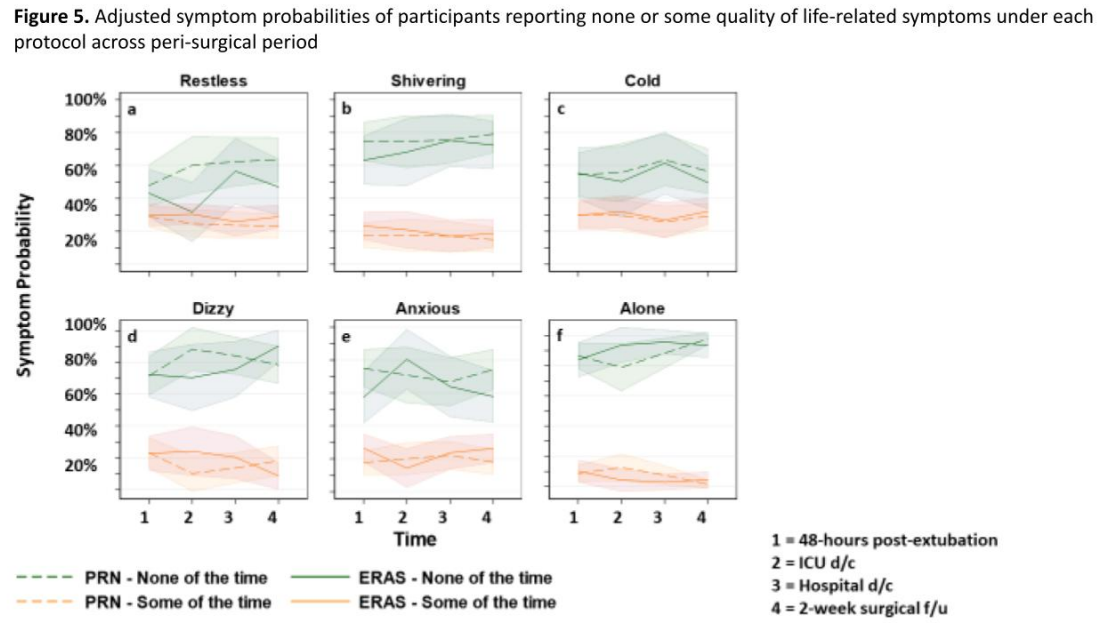


Figure 4. Adjusted symptom probabilities of participants reporting none or some pain-related symptoms under each protocol across peri-surgical period
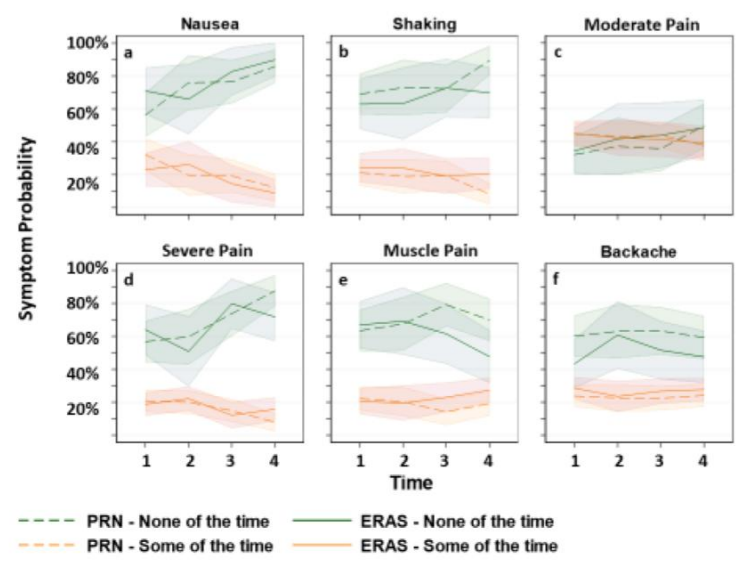

Time

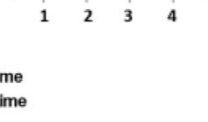

$1=48$-hours post-extubation

$2=I C U d / c$

$3=$ Hospital $d / c$

$4=2$-week surgical $f / u$

\section{ERAS Protocol}

post-oo
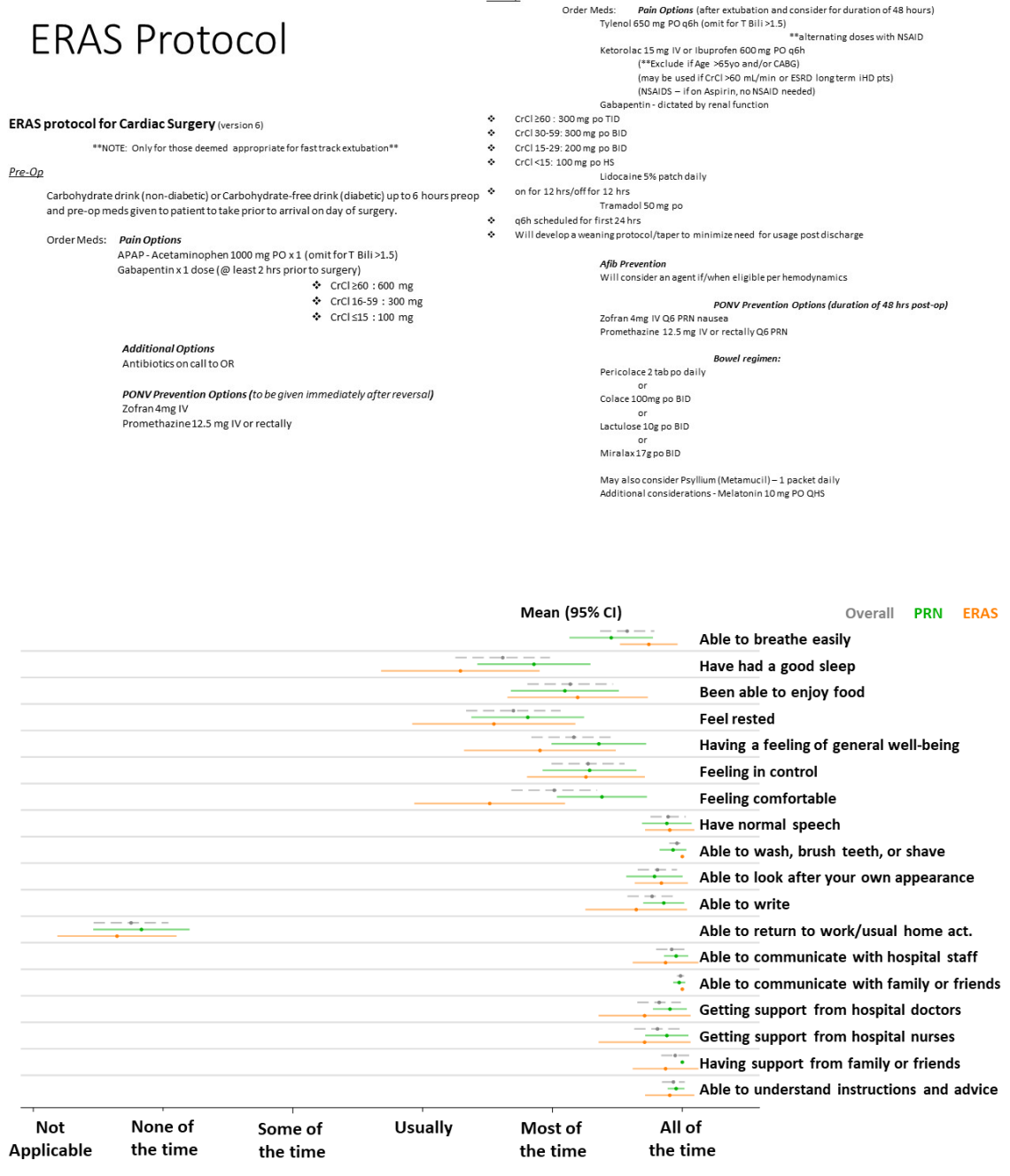


Nausea
Vomiting
Dry-retching
Feeling restless
Shaking or twitching
Shivering
Feeling too cold
Feeling dizzy
Had bad dreams
Feeling anxious
Feeling angry
Feeling depressed
Feeling alone
Had difficulty falling asleep
Feeling confused
$\begin{aligned} & \text { Moderate pain } \\ & \text { Severe pain } \\ & \text { Headache } \\ & \text { Muscle pains } \\ & \text { Backache } \\ & \text { Sore throat } \\ & \text { Sore mouth }\end{aligned}$
$\begin{aligned} & \text { Not } \\ & \text { Applicable }\end{aligned}$

